\title{
AVALIAÇÃO DE CORRELAÇÓES DE DIÂMETRO HIDRÁULICO EM DUTOS ANULARES
}

\author{
Eduardo da Cunha Hora Paraiso ${ }^{11}$ \\ Cláudia Miriam Scheid ${ }^{1}$ \\ Luís Américo Calçada \\ Deividson Silveira dos Santos ${ }^{2}$ \\ Caroline Eulino Gonçalves Pereira ${ }^{3}$ \\ Edilson Adrião Cabral
}

\begin{abstract}
Resumo: Nos projetos hidráulicos de poços de petróleo, os fluidos de perfuração e completação circulam em regióes anulares. Dentre os fatores que influenciam o cálculo da perda de carga, nestas regióes, o diâmetro hidráulico é determinante. $\mathrm{O}$ objetivo deste trabalho foi avaliar as correlaçóes de diâmetro hidráulico utilizando fluidos Newtonianos. Quatro correlaçôes de diâmetro hidráulico foram analisadas e uma metodologia experimental é apresentada para o cálculo do diâmetro hidráulico experimental. Além da análise com fluidos Newtonianos, um estudo de caso é realizado com resultados do escoamento de pasta de cimento, um fluido não-Newtoniano utilizado na cimentação de poços de petróleo. Neste caso a correlação de diâmetro hidráulico desenvolvida é avaliada frente às demais. A correlação experimental apresentou bons resultados no estudo de caso com a pasta de cimento, com erros percentuais absolutos médios entre 9,6 e $16,6 \%$, mostrando-se mais estável, sem extremos elevados como ocorre com as demais.
\end{abstract}

Palavras-chave: perda de carga, escoamento anular, diâmetro hidráulico.

Abstract: During the hydraulic projects of oil wells, the drilling and completion fluids flow in annular regions. Among the factors that influence the pressure loss, in these regions, the hydraulic diameter is decisive. The goal of this research was to evaluate hydraulic diameter correlations using Newtonian fluids. Four hydraulic diameter correlations were analyzed and an experimental methodology is presented to calculate the hydraulic diameter. Besides using Newtonian fluids to analyze the correlations, results of cement slurry flow were employed as a case study. Being the latter, a non-Newtonian fluid used in the process of oil well cementing. In this case, the hydraulic diameter correlation developed is evaluated against the others. The experimental correlation yielded good results in relation to the cement slurry, presenting average errors ranging from 9.6 to $16.6 \%$, showing more stability unlike the other correlations.

Keywords: pressure loss, annular flow, hydraulic diameter.

1Departamento de Engenharia Química da Universidade Federal Rural do Rio de Janeiro.

${ }^{2}$ Discente da Pós-Graduação em Engenharia Química da Universidade Federal Rural do Rio de Janeiro

${ }^{3}$ Discente do curso de Engenharia Química da Universidade Federal Rural do Rio de Janeiro.

Endereço: BR 465, Km 7 - 23.890-000 - Seropédica/RJ e-mail: eduardocunha@ufrrj.br 


\section{INTRODUÇÃO}

O estudo do escoamento de fluidos Newtonianos e não-Newtonianos em dutos é de grande interesse da engenharia. $\mathrm{Na}$ indústria petrolífera, destacam-se os processos de perfuração e cimentação de poços. Nestes processos, ocorre a circulação de fluidos em regióes tubulares, interior da coluna, e anulares, entre a formaçáo rochosa e o exterior do revestimento, Lake e Mitchel (2006).

Em regiôes anulares, para utili-zação das equaçóes tradicionais de escoamento de fluidos, Newtonianos e não-Newtonianos, é necessária a deter-minação de um parâmetro ou fator geométrico capaz de representar a regiáo anular como um duto circular.

Diversos autores têm desenvol-vido correlaçôes, empíricas ou experi- mentais, para os chamados diâmetros hidráulicos ou equivalentes. $\mathrm{O}$ diâmetro hidráulico é usado em cálculos de projeto hidráulico da perfuração e completação de poços (Bourgoyne et al.,1991).

O objetivo deste trabalho foi de avaliar quatro correlaçôes de diâmetro hidráulico utilizando-se dados de queda de pressão e vazão no escoamento de água e glicerina $(98 \% \mathrm{~m} / \mathrm{v})$, a $25^{\circ} \mathrm{C}$, em regióes anulares.

Além disto, dados de queda de pressão do escoamento de água em regiōes anulares são também utilizados no desenvolvimento de uma metodologia para determinação de um diâmetro hidráulico experimental. A partir da metodologia apresentada, uma correlação experimental é desenvolvida.

Esta correlação, bem como as demais analisadas, é utilizada em um estudo de caso na análise do escoamento de pasta de cimento, fluido não-Newtoniano, utilizado no processo de cimentaçáo de poços de petróleo.

\section{REVISÃO DE LITERATURA}

\subsection{BALANÇO DE ENERGIA MECÂNICA NO ESCOAMENTO DE FLUIDOS}

Fox et al. (2004) apresentam a e-quação de Bernoulli modificada que representa o balanço de energia mecânica no escoamento isotérmico e estacionário de um fluido incompressível entre dois pontos quaisquer, neste caso 1 e 2 , onde não há acidentes e sem o fornecimento de energia.

$$
\frac{P_{1}}{\rho g}+\frac{v_{1}^{2}}{2 g}+z_{1}=\frac{P_{2}}{\rho g}+\frac{v_{2}^{2}}{2 g}+z_{2}+b
$$

Onde $h d$ pode ser calculado pela equação de Darcy-Weisbach;

$$
h_{d}=f_{d}\left(\frac{L}{D_{H}}\right) \frac{\langle v\rangle^{2}}{2 g}
$$

O fator de atrito experimental de Fanning ( $f_{\text {exp }}$ $\left.=4 f_{d}\right)$ é oriundo da subs-tituição da Equação 2 em 1 , feitas as consideraçóes citadas.

$$
f_{\text {exp }}=\frac{\Delta D_{H}}{2 \rho L\langle v\rangle^{2}}
$$

Sendo $\Delta \mathrm{P}$ a queda de pressáo $(\mathrm{Pa}), \mathrm{D}_{\mathrm{H}} \mathrm{o}$ diâmetro hidráulico $(\mathrm{m}), \rho$ a densidade do fluido $\left(\mathrm{Kg} / \mathrm{m}^{3}\right)$, L a distância entre as tomadas de pressão $(\mathrm{m})$ e $\langle v\rangle$ a velocidade média de escoamento $(\mathrm{m} / \mathrm{s})$.

Para fluidos Newtonianos, o fator de atrito laminar de Fanning é mostrado em Bird et al. (2002):

$$
f=\frac{6}{R}
$$

Onde;

$$
R=\frac{D\langle v\rangle \rho}{\mu}
$$

\subsection{CORRELAÇÓES DE DIÂMETRO HI- DRÁULICO DE REGIÓES ANULARES}

Quatro equaçôes de diâmetro hi-dráulico foram avaliadas neste trabalho. Ambas funções do diâmetro externo do tubo interno $\left(\mathrm{D}_{1}\right)$, e do diâmetro interno do tubo externo $\left(\mathrm{D}_{2}\right)$ do anular.

A primeira das correlaçôes $\left(\mathrm{DH}_{1}\right)$, foi desenvolvida analiticamente, consi-derando a região anular, formada por dois cilindros concêntricos, como uma fissura formada por duas placas planas. Esta teoria é conhecida como Slot. Essas consideraçôes são válidas quando a rela-ção entre o diâmetro interno e externo é maior que 0,3 (Bourgoyne et al., 1991). Em experimentos de perda de carga realizados com pastas de cimento e fluidos 
de perfuração, Silva et al. (1989) relatam a eficiência desta correlação frente as demais avaliadas.

$$
\boldsymbol{H}_{1}=0,816\left(D_{2}-D_{1}\right)
$$

A segunda equação $\left(\mathrm{DH}_{2}\right)$ foi desenvolvida analiticamente a partir da teoria do raio hidráulico (Bourgoyne et al., 1991). A relação entre a área da seção transversal e do perímetro molhado da região anular determina o diâmetro hidráulico, que é equivalente a quatro vezes o valor do raio hidráulico. Segundo Bourgoyne et al. (1991) esta correlação é a mais utilizada na indústria petrolífera.

$$
\boldsymbol{H}_{2}=4 R_{H}=\left(D_{2}-D_{1}\right)
$$

A terceira equação $\left(\mathrm{DH}_{3}\right)$ foi desenvolvida por Lamb (1945). Esta e-quação foi desenvolvida a partir do critério de obtenção da geometria equivalente decorrente do princípio da medida de perda de carga no regime laminar de fluidos Newtonianos escoando em geometrias tubulares e anulares.

$$
\boldsymbol{H}_{3}=\sqrt{D_{2}^{2}+D_{1}^{2}-\frac{D_{2}^{2}-D_{1}^{2}}{h\left(D_{2} / D_{1}\right)}}
$$

Crittendon (1959) obteve a Equa-ção 9, a partir de um trabalho empírico, através de experimentos com fluidos de fraturamento. Para fins acadêmicos neste trabalho chamaremos de $\mathrm{DH}_{4}$.

$$
\boldsymbol{H}_{4}=\frac{\sqrt[4]{D_{2}^{4}-D_{1}^{4}-\frac{\left(D_{2}^{2}-D_{1}^{2}\right)^{2}}{h\left(D_{2} / D_{1}\right)}}+\sqrt{D_{2}^{2}-D_{1}^{2}}}{2}
$$

\section{MATERIAIS E MÉTODOS}

A unidade experimental utilizada para avaliação das correlaçôes de diâmetro hidráulico (Figuras 1 e 2) con-siste de um sistema que opera em circuito fechado composto de tubos de ferro galvanizado, uma bomba de desloca-mento positivo do tipo helicoidal de $/ 25 \mathrm{HP}$ com vazóes de até $20 \mathrm{~m}^{3} / \mathrm{h}$ e pressôes de recalque da ordem de $12 \mathrm{kgf} / \mathrm{cm}^{2}$.

Transdutores de pressão diferencial fornecem a queda de pressáo em cada arranjo estudado. Um medidor de vazão mássica tipo coriólis fornece as vazóes, temperaturas e densidades do fluido durante o escoamento. O tanque de mistura é dotado de agitação e aquecimento, podendo ainda ser refrigerado por um chiller, com potência de refrigeração de $15.000 \mathrm{kcal} / \mathrm{h}$.

Os trechos de análise são formados por dutos anulares concêntricos com diâmetros externos e internos com respectivamente: Anular I (2" e $11 / 2 ")$, Anular II (1 $1 / 2$ " e 1 ”), Anular III ( 1 1/4" e 3/4"), Anular IV (1 1/2" e 3/4”), Anular V (2” e 1 $1 / 4$ ”), Anular VI (3" e 1 1/4”). Os trechos anulares possuem $3 \mathrm{~m}$ de com-primento e as tomadas de pressão são posicionadas a $1,5 \mathrm{~m}$ da entrada e a $1 \mathrm{~m}$ da saída de maneira a garantir a estabilização do fluxo. Estes anulares foram construídos mantendo-se uma similaridade geométrica com poços de petróleo usuais, através da razão de diâmetro $\left(\mathrm{D}_{2} / \mathrm{D}_{1}\right)$.

Figura 1. Esquema representativo da unidade.

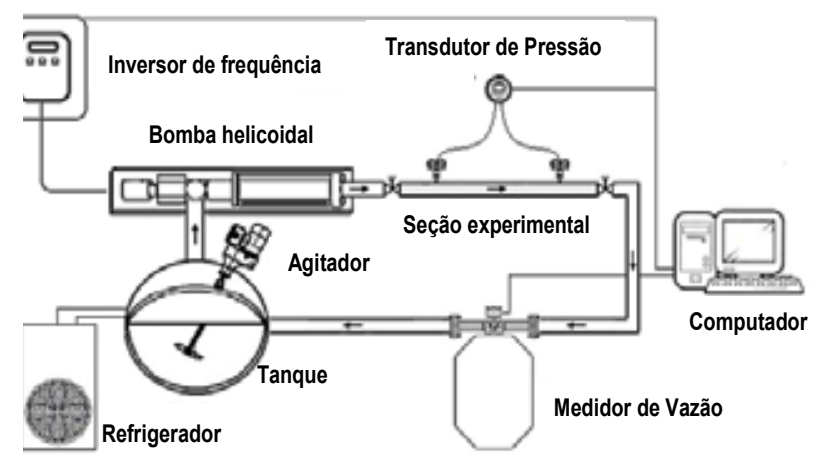

Figura 2. Foto da unidade experimental (1-Tanque; 2- Refrigerador; 3- Bomba helicoidal; 4- Trecho experimental; 5Transdutor de pressão; 6- Medidor de vazão).

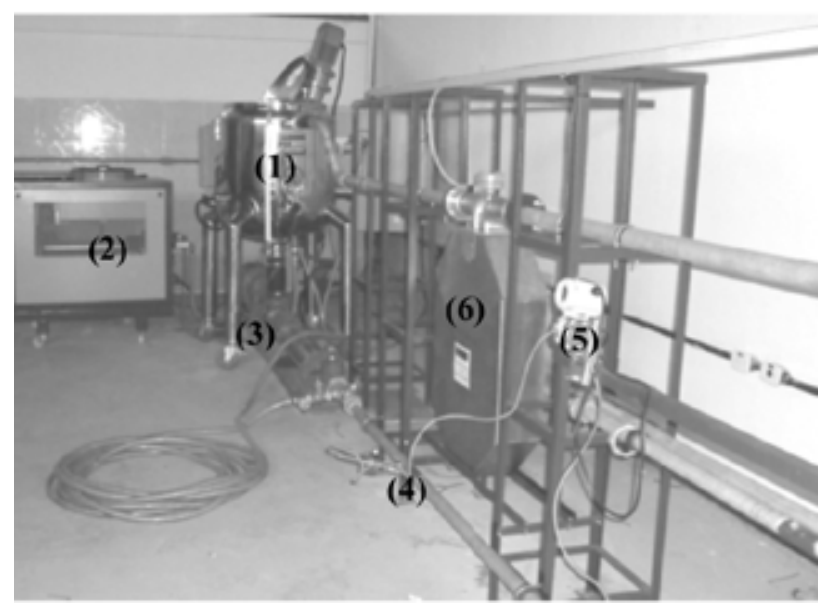

Para avaliação das correlaçôes de diâmetro hidráulico foram utilizados dados de queda de pressão e vazão, no regime laminar, de água nos anulares I a V, e uma solução de glicerina $(98 \%$ 
$\mathrm{m} / \mathrm{v}$ ) nos anulares IV e VI. Em ambos os fluidos a temperatura do teste foi de $25(+/-2)^{\circ} \mathrm{C}$,

Os dados de perda de carga e vazão de água, no regime laminar a $25^{\circ} \mathrm{C}$, nos anulares I a V são utilizados para desenvolvimento da metodologia de cálculo do diâmetro hidráulico expe-rimental.

Um estudo de caso é realizado com resultados de queda de pressão e vazão do escoamento de pasta de cimento, a fim de avaliar a correlaçáo experimental desenvolvida frente às demais.

Os dados reológicos foram obtidos em viscosímetro FANN 35A na temperatura do escoamento do fluido. Foram realizados testes ao longo do experimento em vazóes predeterminadas para avaliar a reologia do fluido com o tempo.

Características dos fluidos e dos dutos estudados estão resumidas na Tabela1.

Tabela 1. Descrição dos fluidos e dutos.

\begin{tabular}{|c|c|c|}
\hline & Água & G licerina $98 \%(\mathrm{~m} / \mathrm{v})$ \\
\hline Composição & Água doce & $\begin{array}{l}\text { Água doce } \\
\text { Glicerina }\end{array}$ \\
\hline Massa especifica & $1000 \mathrm{Kg} / \mathrm{m}^{2}$ & $974-1150 \mathrm{Kg} / \mathrm{m}^{3}$ \\
\hline Vscosidade & $0,925 \propto P$ & $39,2-38,3 \subset P$ \\
\hline Temperatura & $25(+/-2)^{\circ} \mathrm{C}$ & $25(+1-2)^{\circ} \mathrm{C}$ \\
\hline Dutos anulares & $\begin{array}{c}\text { I }\left(2^{m}-11 / 2^{m}\right) \\
\text { II }\left(11 / 2^{\prime \prime}-1^{\prime \prime}\right) \\
\text { III }\left(11 / 4^{\prime \prime}-3 / 4^{\prime \prime}\right) \\
\text { IV }\left(11 / 2^{m}-3 / 4^{\prime \prime}\right) \\
\text { V }\left(2^{m}-11 / 4^{\prime \prime}\right)\end{array}$ & $\begin{array}{l}\text { IV }\left(11 / 2^{\prime \prime}-3 / 4^{\prime \prime}\right) \\
\text { И }\left(3^{\prime \prime}-11 / 4^{\prime \prime}\right)\end{array}$ \\
\hline
\end{tabular}

\section{RESULTADOS E DISCUSSÃO}

\subsection{AVALIAÇÃO DAS CORRELAÇÕES DE DIÂMETRO HIDRÁULICO}

Nesta avaliação, a perda de carga teórica é obtida com a substituição do fator de atrito experimental (Equação 3), na equação do fator de atrito laminar (Equação 4). Na equação resultante é então inserida a correlação de diâmetro hidráulico, que se deseja avaliar, e desta forma é possível o cálculo da perda de carga teórica do trecho, no regime laminar.

Para exemplificar a avaliação realizada, são apresentadas as Figuras 3a e 3b, que são gráficos comparativos da perda de carga experimental, obtida no escoamento de água, a $25^{\circ} \mathrm{C}$, e de Gliceri- na $(98 \% \mathrm{~m} / \mathrm{v})$, ambos no duto Anular IV (1 1/2" e $3 / 4$ ”). Nestas figuras, os valores da queda de pressão foram comparados através do erro percentual absoluto, Equação 10.

$$
\operatorname{Erro}(\%)=\frac{\left|\Delta P_{\text {exp }}-\Delta P_{\text {calc }}\right|}{\Delta P_{\text {exp }}} 100
$$

Os erros percentuais médios dos demais experimentos, bem como os apresentados nas $\mathrm{Fi}$ guras $3 \mathrm{a}$ e 3 b, são sintetizados na Tabela 2 . Nesta tabela os erros percentuais menores que $20 \%$ são destacados.

Analisando a Figuras 3 e a Tabela 2 é possível perceber que nos experimentos realizados com água há uma alternância na eficácia das correlações $\mathrm{DH}_{1}$ e $\mathrm{DH}_{3}$, com a correlação $\mathrm{DH}_{2}$.
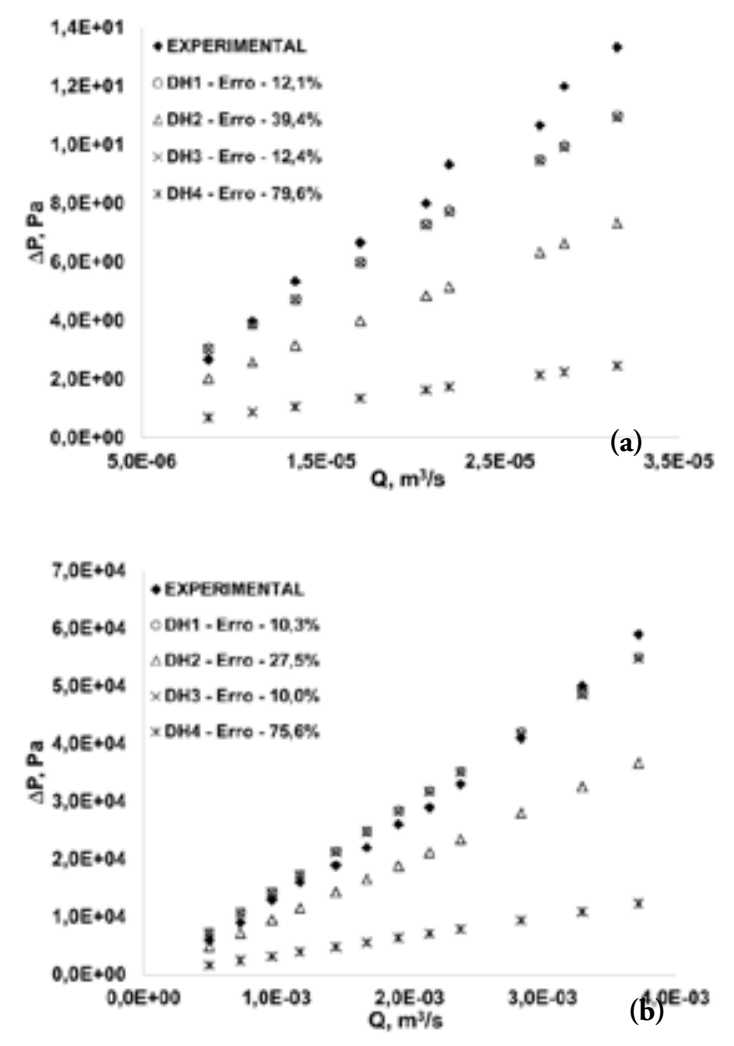

Figura 3. Avaliação das equaçóes de diâmetro hidráulico no duto Anular IV (1 1 1 2 ” e 3/4”), no escoamento: (a) água; (b) Glicerina $98 \% \mathrm{~m} / \mathrm{v}$.

Comparando os resultados dos testes com água e glicerina no anular IV, nota-se que a mudança do fluido não gerou alteração na ordem de eficiência das correlaçóes, levando a crer que esta alternância deva estar relacionada com a área de 
escoamento e com a razão de diâmetro de cada anular.

Tabela 2. Erros percentuais absolutos médios das correlações de diâmetro hidráulico.

\begin{tabular}{|c|c|c|c|c|c|c|c|c|}
\hline \multirow{2}{*}{ Anular } & \multicolumn{4}{|c|}{$\begin{array}{l}\text { Erro percentual absoluto médio } \\
\text { Agua, } 25^{\circ} \mathrm{C}\end{array}$} & \multicolumn{4}{|c|}{$\begin{array}{l}\text { Erro percentual absoluto médio } \\
\text { Glicerina }(98 \% \mathrm{~m} / \mathrm{v}), 25^{\circ} \mathrm{C}\end{array}$} \\
\hline & $\mathrm{DH}_{1}$ & $\mathrm{DH}_{2}$ & $\mathrm{DH}_{3}$ & $\mathrm{DH}_{4}$ & $\mathrm{DH}_{1}$ & $\mathrm{DH}_{2}$ & $\mathrm{DH}_{3}$ & $\mathrm{DH}_{4}$ \\
\hline Anular I $\left(2^{\prime \prime}-1 \frac{11 / 2}{2}\right)$ & 36,7 & 12,6 & 36,5 & 90,3 & - & - & - & - \\
\hline Anular II (1 1/2" - 1") & 9,8 & 26,9 & 9,6 & 86,1 & - & - & - & - \\
\hline Anular III (1 1/4" - 3/4") & 59,1 & 10,7 & 58,7 & 73,9 & - & - & - & - \\
\hline Anular $N\left(1 \frac{1}{2}-3 / 4^{\prime \prime}\right)$ & 12,1 & 39,4 & 12,4 & 79,6 & 10,3 & 27,5 & 10,0 & 75,6 \\
\hline Anular V $\left(2^{\prime \prime}-11^{1 / 4}\right)$ & 17,4 & 21,8 & 17,1 & 83,7 & - & - & - & - \\
\hline Anular VI $\left(3^{\prime \prime}-1^{1 / 1} 4^{\prime \prime}\right)$ & - & - & - & - & 5,6 & 35,0 & 6,0 & 71,1 \\
\hline
\end{tabular}

Em todos os testes realizados, a correlaçáo $\mathrm{DH}_{4}$ apresentou resultado insatisfatório frente às demais.

\subsection{METODOLOGIA PARA CÁLCULO DO DIÂMETRO HIDRÁULICO EXPERIMENTAL $\left(\mathrm{DH}_{\text {EXP }}\right)$}

Com o intuito de melhorar a previsão da perda de carga nas regióes anulares e visando reduzir o erro percentual médio das correlaçóes de diâmetro hidráulico, foi utilizada uma metodologia para o cálculo de um diâmetro hidráulico experimental $\left(\mathrm{DH}_{\exp }\right)$.

$\mathrm{O}$ fator de atrito experimental (Equação 3) foi aproximado ao fator de atrito de Fanning para escoamento La-minar (Equação 4).

$$
f_{\text {exp }} \cong f_{h \min a}
$$

Simplificando:

$$
Q=\frac{B_{\exp }^{2}}{\left(\frac{\boldsymbol{3} \cdot \mu \cdot L}{A}\right)} \Delta P
$$

A partir da Equação 12, com dados experimentais da queda de pressáo e vazão em dutos anulares, no regime laminar de fluidos Newtonianos, é possível calcular o diâmetro hidráulico experimental $\left(\mathrm{DH}_{\text {exp }}\right)$ para cada geome-tria anular avaliada.

Dados experimentais de queda de pressão e vazão, do escoamento de água a $25^{\circ} \mathrm{C}$ no regime laminar, foram selecionados e relacionados graficamente. Para exemplificar a análise realizada é apresentada a Figura 4, onde são utilizados os dados de queda de pressão do anular I.

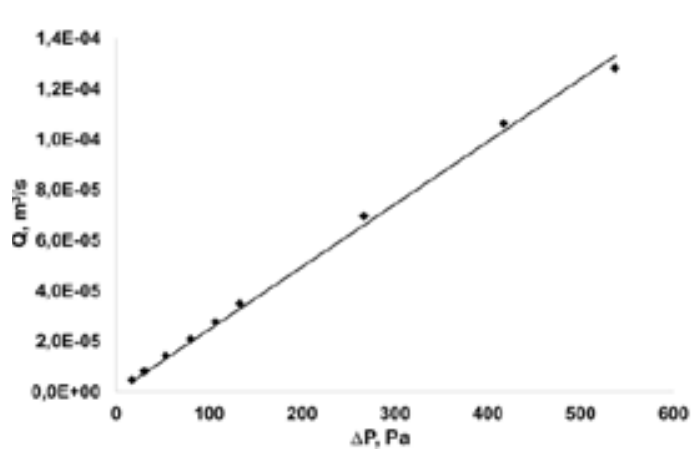

Figura 4. Relação entre vazão e queda de pressão no Anular I ( 2 " $11 \frac{1}{2}$ ”), escoamento laminar.

Os resultados apresentados na Figura 4 demonstram uma boa linearidade dos pontos experimentais. A mesma análise gráfica foi realizada nos demais anulares. Os coeficientes angulares obtidos foram comparados com a Equação 12, a fim de calcular o $\mathrm{DH}_{\text {exp }}$ em cada anular pesquisado. Estes resultados são apresentados na Tabela 3, onde sáo comparados com as demais correlaçóes.

Os valores em destaque na Tabela 3 correspondem aos $\mathrm{DH}_{\text {exp }}$ obtidos em cada anular. De posse destes, foi possível construir a Figura 5 de onde se calcula, através da linha de tendência, a equação que melhor ajusta os pontos experimentais de $\mathrm{DH}_{\text {exp }}$, que será então a correlação do diâmetro hidráulico experimental desenvolvida neste trabalho.

Tabela 3. Resultado do $\mathrm{DH}_{\text {exp }}$ para cada anular e

\begin{tabular}{|c|c|c|c|c|c|}
\hline Anular & $D H_{\exp }, \mathrm{m}$ & $\mathrm{DH}_{1}, \mathrm{~m}$ & $\mathrm{DH}_{2}, \mathrm{~m}$ & $\mathrm{DH}_{3}, \mathrm{~m}$ & $\mathrm{DH}_{4}, \mathrm{~m}$ \\
\hline Anularl $\left(2^{x}-11^{1 / 2}\right)$ & 0,0054 & 0,0046 & 0,0056 & 0,0046 & 0,0172 \\
\hline Anular II $\left(11 R^{\prime \prime}-1^{\prime \prime}\right)$ & 0,0070 & 0,0068 & 0,0084 & 0,0068 & 0,0192 \\
\hline Anular III (1 $\left.1 / 4^{\prime \prime}-3 / 4^{\prime \prime}\right)$ & 0,0092 & 0,0078 & 0,0096 & 0,0078 & 0,0193 \\
\hline Anular IV $\left(11 / 2^{n}-3 / 4^{n}\right)$ & 0,0115 & 0,0124 & 0,0152 & 0,0124 & 0,0262 \\
\hline AnularV $\left(2^{x}-11^{x}\right)$ & 0,0100 & 0,0096 & 0,0118 & 0,0096 & 0,0258 \\
\hline
\end{tabular}
transdutor de pressão.

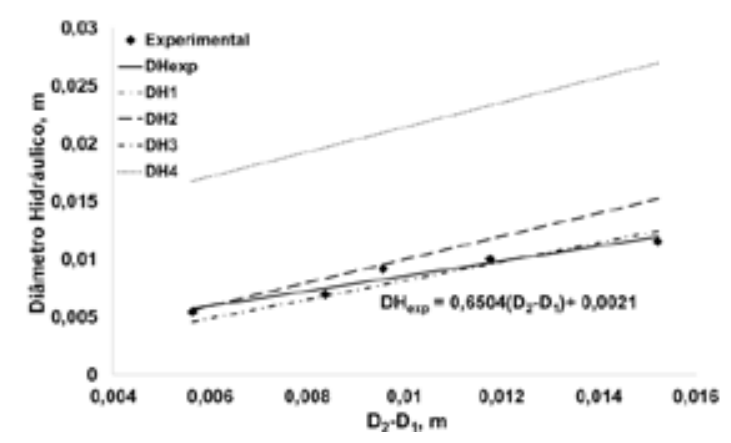

Figura 5. Ajuste e comparação da correlação de diâmetro hidráulico. 
Através da análise da Figura 5, foi possível verificar que a correlação obtida (Equação 13) é capaz de representar bem as regióes anulares estudadas.

$$
\boldsymbol{H}_{\text {exp }}=0,6504\left(D_{2}-D_{1}\right)+0,0021
$$

A metodologia utilizada para desenvolvimento do diâmetro hidráulico experimental mostrou-se satisfatória pela proximidade com as correlaçóes $\mathrm{DH}_{1}$ e $\mathrm{DH}_{3}$, que apresentaram os melhores resultados nos experimentos com a glicerina e ainda pela proximidade com a correlação $\mathrm{DH}_{2}$ que é uma das mais usuais na área de escoamento de fluidos. A correlação $\mathrm{DH}_{4}$ apresentou resultados insatisfatórios.

O distanciamento que as cor-relaçóes apresentam para elevados valo-res de diferença de diâmetro $\left(\mathrm{D}_{2}-\mathrm{D}_{1}\right)$, pode representar uma diferença substancial na previsão da perda de carga em poços de petróleo, já que nestes as diferenças de diâmetro são elevadas. As correlaçóes $\mathrm{DH}_{3}$ e $\mathrm{DH}_{1}$ são equivalentes e os resultados estáo sobrepostos na Figura 5.

Com o intuito de estudar a eficácia da correlação experimental foi necessária sua utilização na previsão da perda de carga de fluidos diferentes de água e glicerina. Para isto, é apresentado a seguir, um estudo de caso com escoamento de pasta de cimento.

\subsection{ESTUDO DE CASO DA AVALIAÇÃO DO DIÂMETRO HIDRÁULICO COM PASTA DE CIMENTO}

A relevância deste estudo de caso está na necessidade de previsão da perda de carga nos processos de cimentação de poços de petróleo. Neste processo, além de escoar em regióes circulares, a pasta de cimento é injetada em regiôes anulares, formada pela formação rochosa perfurada e a parede externa do duto de revestimento.

Com o intuito de avaliar cor-relaçóes de diâmetro hidráulico para o processo de cimentação de poços de petróleo, foram utilizados dados de queda de pressáo e vazáo de pasta de cimento em regiōes anulares.

A pasta de cimento analisada foi formulada pelo CENPES/PETROBRAS e possui características de fluido não-Newtoniano independente do tempo. A pasta de cimento foi produzida no tanque de mistura da unidade experimental sendo composta por cimento classe $\mathrm{G}$, água doce e aditivos (retardador de pega e anti-espumante).

Para este tipo de fluido, a visco-sidade varia com a taxa de deformação. Cada modelo reológico possui uma equação desenvolvida para o fator de atrito no regime laminar.

A Equação 14 apresenta o fator de atrito laminar, desenvolvido para o modelo reológico de Herschell-Buckley. Esta equação foi utilizada na análise da pasta de cimento estudada por apresentar bons resultados com este tipo de fluido (Silva et al., 1989; Paraiso, 2011)

$$
f=\frac{2 H_{H}}{R_{B}^{2}}+\frac{\Phi}{R_{B}}[A]^{-n_{B}}
$$

Onde;

$$
\begin{aligned}
& {[A]=\left[\begin{array}{l}
\left(1-\frac{E_{H}}{\tau_{w}}\right)^{3}+\frac{2\left(3 n_{H}+1\right)}{\left(2 n_{H}+1\right)}\left(\frac{E_{H}}{\tau_{w}}\right)\left(1-\frac{E_{H}}{\tau_{w}}\right)^{2}+ \\
\frac{\left(3 n_{H}+1\right)}{\left(n_{H}+1\right)}\left(\frac{E_{H}}{\tau_{w}}\right)^{2}\left(1-\frac{E_{H}}{\tau_{w}}\right)
\end{array}\right]} \\
& \mathbb{R}_{H}=\frac{8 D^{n_{H}}\langle v\rangle^{2-n_{H}} \rho}{k_{H}\left[2\left(3 n_{H}+1\right) / n_{H}\right]^{n_{H}}} \\
& H_{H}=R_{H}^{2} \frac{E}{\rho\langle v\rangle^{2}}
\end{aligned}
$$

$$
\frac{\boldsymbol{E}_{\mathbb{H}}}{\tau_{w}}=2 \frac{\boldsymbol{H}_{\boldsymbol{H}}}{f \mathrm{R}_{\boldsymbol{H}}^{2}}
$$

Neste caso, a perda de carga teórica é obtida com a substituição do fator de atrito experimental (Equação 3), na equação do fator de atrito laminar desenvolvido para o modelo reológico de Herschell-Buckley (Equação 14). Na e-quação desenvolvida é inserida a correlação de diâmetro hidráulico, que se deseja avaliar, e então é possível o cál-culo da perda de carga teórica do trecho, no regime laminar.

Para ilustrar os resultados obtidos é apresentada a Figura 6, onde são com-paradas as perdas de carga experimental e calculadas, obtidas no escoamento da pasta de cimento no Anular III ( $1 \frac{1 / 4}{4}$ e $\left.3 / 4 "\right)$, a $25^{\circ} \mathrm{C}$. Nesta figura, os valores de queda de pressão experimentais e teóricas são comparadas através do erro percentual, Equação 9. Os erros per-centuais absolutos médios das correlaçóes de diâmetro hidráulico nos Anulares I, II e III, a $25^{\circ} \mathrm{C}$, foram condensados na Tabela 4 . 


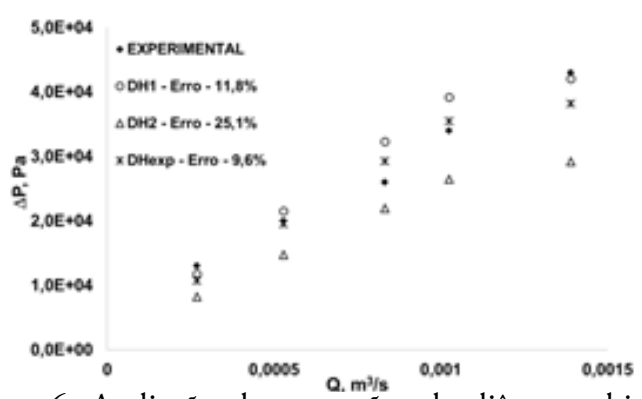

Figura 6. Avaliação das equaçốes de diâmetro hidráulico para pasta de cimento no anular III $\left(1 \frac{1 / 4}{4} \mathrm{e}^{3 / 4} 4^{\prime}\right)$ a $25^{\circ} \mathrm{C}$.

Tabela 4. Erros percentuais médios das correlaçóes de diâmetro hidráulico.

\begin{tabular}{|c|c|c|c|}
\hline \multirow{2}{*}{ Anular } & \multicolumn{3}{|c|}{ Erro percentual absoluto médio } \\
\hline & $\mathrm{DH}_{1}$ & $\mathrm{DH}_{2}$ & DHexp \\
\hline Anular I (2"- 1 1/2") & 59,1 & 15,5 & 16,6 \\
\hline Anular II (1 1/2" - 1") & 11,9 & 27,1 & 9,8 \\
\hline Anular III (1 1/4" - 3/4") & 11,8 & 25,1 & 9,6 \\
\hline
\end{tabular}

A análise da Figura 6 e da Tabela 4 mostra que as correlaçóes $\mathrm{DH}_{1}$ e $\mathrm{DH}_{2}$ apresentam bons resultados em casos separados, no entanto, é possível perceber que a mudança na geometria causa uma alternância na eficácia destas correlaçóes.

A correlação $\mathrm{DH}_{\text {exp }}$, no entanto, mostra-se mais estável, sem valores de erros percentuais extremos, como ocorre com as demais com a substituição da geometria.

\section{CONCLUSÁO}

$\mathrm{Na}$ avaliação do diâmetro hidráulico, com solução de glicerina a $98 \% \mathrm{~m} / \mathrm{v}$, as correlaçóes $\mathrm{DH}_{1}$ e $\mathrm{DH}_{3}$ apresentam resultados matematicamente equivalentes. Estas apresentam ainda os menores erros percentuais médios, con-forme mostrado na Tabela 2.

Nos experimentos realizados com água há uma alternância na eficácia das correlaçôes $\mathrm{DH}_{1}$ e $\mathrm{DH}_{3}$, com a correlação $\mathrm{DH}_{2}$. Esta alternância possivelmente esta relacionada com a área de escoamento e com a razão de diâmetro de cada anular.

Nos testes realizados com água e glicerina a correlação $\mathrm{DH}_{4}$ apresentou resultados insatisfatórios frente às demais, possivelmente por ter sido origi-nalmente utilizada com fluidos de fraturamento.

O estudo de caso apresentado com dados do escoamento de pasta de cimento mostrou que a correlação de diâmetro hidráulico desenvolvida, apresenta resultados satisfatórios e estáveis frente à mudança de geometria, já que esta apresentou, na maioria dos casos os menores erros percentuais, como pode ser visto na Tabela 4 .

Para que a correlação experi-mental desenvolvida venha ter uma maior validade e aceitação acadêmica, se faz necessário novos testes com diferentes fluidos e outros dutos anulares de diferentes diâmetros hidráulicos.

\section{AGRADECIMENTOS}

Os autores agradecem ao Centro de Pesquisa da Petrobras (CENPES), Coordenação de Aperfeiçoamento de Pessoal de Nível Superior (CAPES) e Conselho Nacional de Desenvolvimento Científico e Tecnológico (CNPq), pelo apoio técnico e financeiro.

\section{NOMENCLATURA}

\begin{tabular}{|c|c|}
\hline A & área de escoamento $\left(\mathrm{m}^{2}\right)$ \\
\hline $\mathrm{D}$ & diâmetro (m) \\
\hline$D_{1}$ & $\begin{array}{l}\text { diâmetro externo do tubo interno do } \\
\text { anular }(\mathrm{m})\end{array}$ \\
\hline$D_{2}$ & $\begin{array}{l}\text { diâmetro interno do tubo externo do } \\
\text { anular }(\mathrm{m})\end{array}$ \\
\hline$D H$ & diâmetro hidráulico (m) \\
\hline$f$ & $\begin{array}{l}\text { fator de atrito de fanning } \\
\text { (adimensional) }\end{array}$ \\
\hline$f_{d}$ & $\begin{array}{l}\text { fator de atrito de Darcy-Weisbach } \\
\text { (adimensional) }\end{array}$ \\
\hline$f_{\text {exp }}$ & $\begin{array}{l}\text { fator de atrito experimental de } \\
\text { fanning (adimensional) }\end{array}$ \\
\hline$f_{\text {laminar }}$ & $\begin{array}{l}\text { fator de atrito laminar de fanning } \\
\text { (adimensional) }\end{array}$ \\
\hline$g$ & aceleração da gravidade $\left(\mathrm{m} / \mathrm{s}^{2}\right)$ \\
\hline hd & perda de carga distribuida $(\mathrm{m})$ \\
\hline$H e_{H B}$ & $\begin{array}{l}\text { número de Hedstron - modelo de } \\
\text { Herschel-Buckley (adimensional) }\end{array}$ \\
\hline
\end{tabular}




\begin{tabular}{|c|c|}
\hline$k_{H B}$ & $\begin{array}{c}\text { índice de consistência para o modelo } \\
\left.\text { de Herschel-Buckley (Pa.s }{ }^{\mathrm{n}}\right)\end{array}$ \\
\hline$L$ & comprimento da seção reta $(\mathrm{m})$ \\
\hline$L E_{H B}$ & $\begin{array}{l}\text { limite de escoamento para fluidos } \\
\qquad(\mathrm{Pa})\end{array}$ \\
\hline$n_{H B}$ & $\begin{array}{l}\text { índice de comportamento do } \\
\text { modelo de Herschel-Buckley }\end{array}$ \\
\hline$P$ & pressão $(\mathrm{Pa})$ \\
\hline$Q$ & vazão de escoamento $\left(\mathrm{m}^{3} / \mathrm{s}\right)$ \\
\hline$R_{H}$ & raio hidráulico $(\mathrm{m})$ \\
\hline$R e_{H B}$ & $\begin{array}{l}\text { número de Reynolds - modelo de } \\
\text { Herschel-Buckley (adimensional) }\end{array}$ \\
\hline$<v>$ & velocidade média $(\mathrm{m} / \mathrm{s})$ \\
\hline$v$ & velocidade (m / s) \\
\hline$z$ & altura $(\mathrm{m})$ \\
\hline
\end{tabular}

Letras e símbolos gregos

$D P \quad$ queda de pressão $(\mathrm{Pa})$

$D P_{\exp } \quad$ queda de pressão experimental $(\mathrm{Pa})$

$D P_{\text {calc }} \quad$ queda pressão calculada para correlação $(\mathrm{Pa})$

$r \quad$ densidade $\left(\mathrm{kg} \cdot \mathrm{m}^{-3}\right)$

$m \quad$ viscosidade dinâmica $\left(\mathrm{kg} \cdot \mathrm{m}^{-1} \cdot \mathrm{s}^{-1}\right)$

$t_{w} \quad$ parâmetro da equação 14 e $17(\mathrm{~Pa})$

\section{REFERÊNCIAS}

Bird, R.B., Stewart, W.E., Lightfoot, E.N. Tranport Phenomena. Second edition, John Wiley \& Sons Inc., 2002.

Bourgoyne Jr, A.T., Millheim, K.K., Chenevert, M.E., YougJr, F.S. Applied drilling engineering. Second printing, Society of petroleum engineers, Richardson, Texas, 1991.

Crittendon, B. C. The mechanics of design and interpretation of hydraulic fracture treatments. Journal of Petroleum Technology, October, pp. 219, 1959.

Fox, R. W., McDonald, A. T., Pritchard, P. J. Introduction to Fluid Mechanics, Six Edition, John Wiley \& Sons Inc., 2004.

Lake L. W., Mitchel R.F. Petroleum Engineering Handbook, v. 2, chapter 3, Society of Petroleum Engineers, 2006.

Lamb, H. S. Hydrodynamics, New York, Dover Publications, pp. 586-187, 1945.

Paraiso, E. C. H. Estudo do escoamento de pastas de cimento em dutos circulares e anulares concêntricos. Dissertação de mestrado, Universidade Federal Rural do Rio de Janeiro, 2011.

Silva, M. G. P. Avaliação de equações pertinentes aos projetos hidráulicos com fuidos de perfuração, pastas de cimento e fluidos de completação no escoamento tubular e anular, Relatório Técnico Interno 67512009,Vol.1, CENPES/ PETROBRAS, 1989. 